\title{
Unübersichtlichkeiten in der Forschungslandschaft
}

\section{Neue Aufgaben und alte Probleme einer Wissenschaftspolitik}

\section{Andreas Knie und Dagmar Simon}

Es wird dieser Tage viel geklagt in der Wissenschaft. Insbesondere in der deutschen Forschungslandschaft hört man zunehmend kritische Stimmen, die sich über die immer umfangreicheren Evaluationen, Kontrollen und Berichtspflichten beschweren. Darüber hinaus ist von einer „Verbetriebswissenschaftlichung der Forschung" die Rede oder gar von einer immer umfassender wirksam werdenden Ökonomisierung des Hochschulwesens, die den Kern des wissenschaftlichen Austausches, den freien und vertraulichen Diskurs mit den Fachkollegen immer beschwerlicher mache (Münch 2007). Andrew Jamison hat im ersten Heft 2008 dieser Zeitschrift dargelegt, dass dieses Gefühl offenkundig auch ein in Europa generell verbreitetes Unbehagen darstellt. Gemeinsam mit Alfred Nordmann ist sich Jamison darin einig, dass damit die Wissenschaft in Gefahr gerät, ihre moralische Integrität als kritisch-reflexive Instanz im Prozess der gesellschaftlichen Modernisierung zu verlieren. Ähnlich argumentieren auch die beiden Ökonomiehistoriker Philip Mirowski und Esther Mirjam Sent im neuen Handbook of Science and Technology Studies: Unter der Herrschaft des „New Public Management" würden die Denk- und Handlungsformen der Wissenschaft an unternehmerische Produktionsformen angepasst und verlören damit ihre zur marktkapitalistischen Verfasstheit des Wirtschaftsraumes komplementären Funktionen (Mirowski/Sent 2007). Diese vorwiegend von Vertretern der geistes- und sozialwissenschaftlichen Disziplinen eingenommenen kritischen Positionen bleiben ihrerseits allerdings nicht ohne kritische Resonanz, denn das Beharren auf Einsamkeit und Freiheit als zentrale Voraussetzung für eine produktive Arbeitsumgebung reicht heute als Rechtfertigung für diesen privilegierten Status kaum noch aus.

Die neuen Formen der Wissensproduktion scheinen sich durch hoch vernetzte, vertikal tief gestaffelte und rekursiv angelegte Kooperationsformen auszuzeichnen, die gerade auch die institutionelle Ordnung der Wissenschaftslandschaft mit ihren rhetorisch nahezu perfekten Arbeitsteilungen 
zu verändern beginnen: Max-Planck-Institute gründen mit Universitäten neue Einrichtungen zur Doktorandenausbildung oder gehen Bündnisse zur kommerziellen Verwertung neuer Erkenntnisse ein; die Deutsche Telekom schließt ihre Zentraleinheit Forschung und erfindet diese als An-Institut an einer Technischen Universität völlig neu; Universitäten und Einrichtungen der außeruniversitären Großforschung planen - angeregt durch die „Exzellenzinitiative“ - sogar komplette Fusionen bis hin zu völlig neuen Supereinheiten, die die bislang geltenden (rechtlichen) Rahmenbedingungen empfindlich berühren.

Es herrscht daher ohne Zweifel eine große Unübersichtlichkeit und Unsicherheit in der Wissenschaftslandschaft, die auch die Frage nach Akteuren, Konzepten, Grenzen und Herausforderungen in und für die Wissenschaftspolitik auf die Agenda der Tagespolitik setzt.

\section{Ent-grenzte Wissenschaft}

Über Indizien, dass sich Form und Inhalt wissenschaftlicher Arbeit verändern, wird in der Wissenschaftsforschung seit längerem debattiert (Nowotny/Scott/Gibbons 2001, Etzkowitz 2002). Zu diesen diskutierten Veränderungen gehört auch die Annahme, dass heutzutage die Produktion von Wissen in räumlich und zeitlich verteilten Prozessen organisiert wird (Mode 2). Es wird davon ausgegangen, dass die Orientierung an den Disziplinen nachlässt und demzufolge das an den Universitäten klassischerweise gelehrte und produzierte disziplinenorientierte Wissen (Mode 1) durch andere Formen wie inter- und transdisziplinäres Wissen und durch die Einflussnahme von gesellschaftlichen Akteuren relativiert wird. Die anschließende Debatte mündet im vorläufigen Konsens, demzufolge beide Produktionstypen immer schon eine Koexistenz führten. Wir wissen aus wissenschafts- und technikhistorischen Studien, dass diese Verbindung zwischen den Forschungstypen von Mode 1 und Mode 2 insbesondere in Deutschland eine lange Tradition hat. Die Namen Carl von Linde, Rudolf Diesel, Carl Bosch oder Hugo Junkers etwa sind nur eine kleine Auswahl von diesen „Wissenschaftsunternehmern", die sich durch eine Doppelkarriere in Wissenschaft und Wirtschaft ausgezeichnet haben. Ebenfalls ist man sich mittlerweile darüber einig, dass Wissenschaft schon immer mehr oder weniger als Teil einer ausdifferenzierten gesellschaftlichen Praxis zu begreifen ist. Gesellschaft, Technik und Wissenschaft durchlaufen ko-evolutionäre Prozesse, die bedeuten, dass gesellschaftliche Dimensionen in die Wissenschaft integriert sind und somit das gesellschaftliche Transformationspotential der Wissenschaft in erheblichem Ausmaß steigern, um „sozial robustes“ Wissen zu erzeugen.

Neu ist aber, dass die institutionelle Trennung zwischen einer abgeschotteten Grundlagenforschung und ihren unterschiedlichen und vielfältigen ge- 
sellschaftlichen Anwendungsbereichen aufgeweicht ist und dass sich dieses "Cross-Over" zu einer akzeptierten wissenschaftlichen Praxis etabliert hat (Weingart/Carrier/Krohn 2007, Etzkowitz 2002). Mittlerweile ist eine Reihe von empirischen Untersuchungen veröffentlicht worden, die die These bestätigen, dass wissenschaftliche Qualität, gemessen an Veröffentlichungen in hochkarätigen refereed journals und wirtschaftlichen Aktivitäten (die unter anderem in Patentanmeldungen und Unternehmensgründungen münden), durchaus positiv korrelieren. Die Verschiebungen im Wertekanon, die ökonomische Handlungen mit wissenschaftlichen Interessen kompatibel machen, treffen zwar nicht gleichermaßen für alle Disziplinen und Fächergruppen $\mathrm{zu}$, scheinen aber insgesamt ein stabiles und verbreitetes Merkmal wissenschaftlicher Produktionsweise zu sein (Zucker u.a. 2006, Bercovitz/ Feldmann 2006).

\section{Be-grenzte Wissenschaftspolitik}

Dass es diese Phänomene der Entgrenzung gibt, darin ist sich die empirische Wissenschaftsforschung weitgehend einig. Strittig sind allerdings die Reichweite dieser Verbindungs- und Hybridisierungsformen sowie die genauen Auswirkungen auf die epistemischen Praktiken sowie auch auf die wissenschaftsinternen Bewertungsprozesse. Wird die wissenschaftliche Neugier, die Kreativität und Ausdauer unterstützt oder drohen hier Gefahren einer viel zu frühen Integration wissenschaftlicher Erkenntnisprozesse in die Verwertungslogiken der Märkte? Welche Auswirkungen haben diese neuen Formen wissenschaftlicher Produktionsweise für die Wissenschaftspolitik? Den Grad der Entgrenzung gilt es daher noch genauer zu ermitteln. Ebenso gilt es, die Auswirkungen auf die wissenschaftliche Qualität zu verstehen. Und genau in diesem Spannungsbogen stellen sich auch die Aufgaben der Wissenschaftspolitik neu.

Grundsätzlich muss hier, anders als in anderen Politikbereichen, eine hohe Autonomie und Eigenständigkeit des Gegenstandsbereiches respektiert werden. Wissenschaft braucht einerseits die Alimentierung durch öffentliche Mittel sowie auch Schutzrechte vor Intervention und ist daher in besonderem Maße auf staatliche Garantien und Finanzleistungen angewiesen. Dies gilt für die Erarbeitung von Reflektionswissen genauso wie für anwendungs- und produktbezogene Erkenntnisse. Gleichzeitig sind aus der Perspektive staatlicher Gewährleistung Rahmenbedingungen zu setzen, damit Quantität und Qualität sowohl der Forschung als auch der Ausbildung des wissenschaftlichen Nachwuchses den gesellschaftlichen Bedürfnissen entsprechen. Die Einflussmöglichkeiten staatlicher Politik sind aber vergleichsweise begrenzt; die Ministerien können Themen und Inhalte nur an die Wissenschaft „delegieren“ und nicht verordnen. Hieraus begründet sich 
das für dieses Politikfeld so typische hohe Maß an Aushandlungs- und Koordinierungsprozessen. Die angedeuteten Entgrenzungsphänomene sowie die Bedeutungszunahme transnationaler Forschungsräume wirken zusätzlich komplexitätssteigernd. Insofern stellt die in der Steuerungs- und Regulierungsforschung (Governanceforschung) beschriebene Zunahme an hybriden Organisationsformen und Koordinierungsmodi auch eine Herausforderung für die Wissenschaftspolitik dar (Braun/Guston 2003).

Eine Grundfrage ist dabei, welches ordnungspolitische Grundverständnis sich Wissenschaftspolitik künftig zu Eigen macht. Soll Wissenschaft weiterhin als eine besondere Form der Erkenntnisproduktion verstanden werden, die zu dem eines besonderen staatlichen Schutzes bedarf? Und lässt sich daraus zweitens auch eine besondere Vorsorgepflicht des Staates ableiten? Dabei ist der Umstand, Wissenschaft einerseits den Sonderstatus eines selbstreferentiellen Raumes zuzugestehen, sie andererseits aber zunehmend mit mangelndem Vertrauen in ihre Leistungsfähigkeit zu konfrontieren, der eigentliche Grund für die vielfältigen und sich oft widersprechenden Governanceformen in der Wissenschaftspolitik. Den ent-grenzten Formen der Wissensproduktion fehlen die entsprechenden institutionellen Unterstützungsstrukturen. Es dominiert der Wunsch seitens der staatlichen Politik nach Kontrolle, ohne über das notwendige Verständniss der wissenschaftlichen Interna zu verfügen. Mehr und mehr werden Methoden und Instrumente marktförmig angelegter Qualitätssicherung eingeführt, die aber den institutionellen Rahmen der öffentlich-rechtlichen Wissenschaftsverfassungen bislang nicht ernsthaft tangieren. Sie entsprechen unterschiedlichen und sich zum Teil widersprechenden Handlungsabsichten und führen zu inkonsistenten Organisations-, Verfahrens- und Regulierungsstrukturen. Externe Kontroll-, Überwachungs- und Koordinierungsansprüche stehen etwa innerwissenschaftlichen Qualitätsstandards gegenüber und lassen ein „Zielwirrwarr“ entstehen. Ähnlich geraten moderne Methoden der Kostenund Leistungsrechnung mit haushaltsrechtlichen Bestimmungen der Kameralistik in Konflikt, Elemente der Ziel- und Leistungsvereinbarungen mit den Bestimmungen des Beamtenrechtes oder marktförmige Leistungsanreize mit ständischen Elementen einer Professionsordnung. Steuerungsparadoxien mit nicht unerheblichen Nebenwirkungen für die betroffenen Lehrund Forschungseinrichtungen sind oftmals die Folge. Die Wissenschaftsadministratoren wissen, dass sie nicht in die Kernbereiche wissenschaftlicher Arbeit unmittelbar eingreifen können und bemühen sich, die institutionelle Gewährleistung für einen „freien“ Wissenschaftsbetrieb durch vielfältige indirekte Steuerungsinstrumente zu organisieren. Die jedoch laufen den Arbeitsweisen und Sinnstrukturen der Wissenschaft vielfach zuwider. Insgesamt sind die Anforderungen einer in bestimmten Feldern und Bereichen ausdifferenzierten und ent-grenzten Wissenschaft in Bezug auf adäquate Formen der Regulierung und Steuerung durch die Wissenschaftspolitik bis- 
lang weitgehend ungeprüft, neue Perspektiven auf die Wissenschaft in unterschiedlichen Kontexten und in verschiedenen Phasen wenig bedacht.

\section{Neue Formate der Wissensproduktion}

Doch wie muss eine dem Produktionsprozess der Wissenschaft angemessene Governanceform aussehen? Sind etwa Definitionsverschiebungen in Bezug auf die Gewährleistungs-, Finanzierungs- und/oder Produktionsverantwortung des Staates notwendig und wenn ja welche? Oder in Begriffen der Wohlfahrtsökonomie ausgedrückt: Benötigen wir eine Neuverständigung über den Status von Wissenschaft als öffentliches Gut? Allerdings ändert sich mit den beschriebenen Hybridisierungen und Entgrenzungen substanziell der Gegenstand, möglicherweise auch die Gestaltungskompetenz und Legitimation der Wissenschaftspolitik. Wissenschaftliches Wissen wird über die institutionellen Grenzen des offiziellen Wissenschaftssystems hinaus produziert, entsprechend ist auch die Rolle der Politik an den „Rändern der Wissenschaft" neu zu prüfen. Gesucht sind Formen der Regulierung und Steuerung, die phasen- und kontextgerecht den wissenschaftlichen Erkenntnisprozess begleiten und angemessen, im Sinne der Schaffung kreativer Arbeitsumgebungen unterstützen können (Knie/Simon 2008). Hier bieten sich neue Herausforderungen für gegenwartsbezogene wie historische Analysen von Wissenschaft. Dabei sollte die Beschäftigung mit der Wissenschaftspolitik weniger sporadisch im Sinne eines politischen Zuständigkeitsbereichs erfolgen, sondern stattdessen das Verhältnis von Wissenschaft und Politik mehr aus einer differenzierungs- oder entdifferenzierungstheoretischen Perspektive (im Zusammenhang mit soziologischer Theoriebildung) beleuchtet werden (Potthast 2009). Gerade durch die historische Analyse der Koproduktion und der -evolution von Wissenschaft und staatlicher Politik bietet die Wissenschaftsforschung aber gute Voraussetzungen, die Möglichkeiten und Grenzen der Wissenschaftspolitik neu zu diskutieren. Dies ist insbesondere dann notwendig, wenn konventionelle Instrumente an den Systemgrenzen wegen institutioneller Neukonstellationen und einer sich verändernden wissenschaftlichen Produktionsweise ihre Bedeutung und ihre Wirkung verlieren.

Wenn es stimmt, dass die Überlappung der Erkenntnisse, die Verfolgung einer wissenschaftlichen Ideen-Validierung durch Nutzungskontexte mit Rückwirkungen auf die Erkenntnisqualität ein Kernelement neuer Wissensproduktion darstellt, dann sind die bestehenden institutionellen Landschaften hierfür nicht vorbereitet und die bisherigen Reformansätze nicht weit genug justiert. Oft führten sie dagegen zu widersprüchlichen Ergebnissen wie beispielsweise die Versuche zur Etablierung einer unternehmerischen Wissenschaft in kameralistisch geprägten Hochschulen. Im Zentrum einer 
neuen Wissenschaftspolitik wird vermutlich daher die Anerkennung eines neuen Formats der Erkenntnisgewinnung liegen. Gesucht wird ein neuer "organisatorischer" Typ der Wissenschaftsproduktion, in dem verschiedene „Welten“ ihre jeweils angemessenen Funktionsräume erhalten, in dem aber auch "Transfer- und Übergangsräume“ fest eingebaut und als soziale Praxis etabliert sind. Neben Lehre und Forschung tritt entrepreneurship als Bestandteil dieses Typs hinzu. Idealerweise werden dann analytische und synthetische, strategische und operative Handlungsformen kombiniert und verbindlich aufeinander bezogen, die dann zu einer neuen gegenseitigen Anerkennungskultur beitragen könnten. Den eingangs erwähnten Mahnungen von Jamison und Nordmann zum Fortbestand einer kritischen Reflexion des Erkenntnisprozesses würden auf diese Weise neue Möglichkeiten geboten.

\section{Literatur}

Bercovitz, Janet/Maryann Feldmann, 2006. Entrepreneurial Universities and Technology Transfer. A Conceptual Framework for Understanding Knowledge-Based Economic Development. Journal of Technology Transfer, 31, 175-188.

Braun, Dietmar/ Guston, David H., 2003. Principal-Agent Theory and Research Policy. An Introduction. Science and Public Policy, 30, 304-307.

Etzkowitz, Henry, 2002. MIT and the Rise of Entrepreneurial Science. London: Routledge.

Knie, Andreas/Simon, Dagmar, 2008. Peers and Politics in der Audit Society. In: Gunnar Folke Schuppert und Andreas Voßkuhle, Hg., Governance von und durch Wissen. Baden-Baden: Nomos, 173-185.

Mirowski, Philip/Sent, Esther-Mirjam, 2007. The Commercialization of Science and the Response of STS. In: Edward Hackett u.a., Hg., The Handbook of Science and Technology Studies. Thousand Oaks/London: Sage, 635-689.

Münch, Richard, 2007. Die akademische Elite. Frankfurt: Suhrkamp.

Nowotny, Helga/Scott, Peter/Gibbons, Michael, 2001. Re-Thinking Science, Knowledge and the Public in an Age of Uncertainty. Cambridge: Polity.

Potthast, Jörg, 2009 [im Druck]. Science and Technology Studies. In: Stefan Hornbostel, Andreas Knie und Dagmar Simon, Hg., Handbuch Wissenschaftspolitik. Wiesbaden: VS Verlag für Sozialwissenschaften.

Weingart, Peter/Carrier, Martin/Krohn, Wolfgang, 2007. Nachrichten aus der Wissensgesellschaft. Analysen zur Veränderung der Wissenschaft. Weilerswist: Velbrück.

Zucker, Lynne G./Darby, Michael R./Furner, Jonathan/Liu, Robert C./Ma, Hongyan, 2007. Minerva Unbound. Knowledge Stocks, Knowledge Flows and New Knowledge Production. Research Policy, 36, 850-863.

Andreas Knie und Dagmar Simon

Wissenschaftszentrum Berlin für Sozialforschung

Forschungsgruppe Wissenschaftspolitik

Reichpietschufer 50

D-10785 Berlin

E-Mail:knie@wzb.eu

E-Mail: dsimon@wzb.eu 